\title{
NUMERICAL SOLUTION FOR FOKKER-PLANCK EQUATIONS IN ACCELERATORS
}

\author{
M.P. Zorzano, H. Mais, DESY, D-22607 Hamburg, Germany and \\ L. Vazquez, Universidad Complutense, 28040 Madrid, Spain
}

\section{Abstract}

A finite difference scheme is presented to solve the FokkerPlanck equation in $(2+1)$ variables numerically. This scheme is applied to study stochastic beam dynamics in two-dimensional phase space.

\section{INTRODUCTION}

One important problem of accelerator physics is to investigate the particle motion under the influence of noise [1]. There are various sources of noise: $r f$ noise, random power supply ripple, random ground motion, restgas scattering, and quantum fluctuations due to radiation. The physical questions one wants to answer are: what is the longtime behaviour of the dynamics, what is the probability for the particle to hit the vacuum chamber (and then be lost) (mean first passage time), what are the average fluctuations of the particle around the periodic design orbit of the accelerator (moments), and what is the time evolution of the probability density (transient and stationary behaviour).

Mathematically stochastic systems can be modelled by stochastic maps (in the time discrete case) and by stochastic differential equations (s.d.e.) in the time continuous case. In the following we will restrict our considerations to s.d.e. with Gaussian white noise. Gaussian white noise is a very good approximation in many accelerator problems [1]. The solution of these s.d.e. are Markovian diffusion processes which can be described by the Fokker-Planck equation [2]. The Fokker-Planck equation is a partial differential equation for the probability density and the transition probability of these stochastic processes.

In general, the stochastic equations of motion of a particle in an accelerator are very complicated and can not be solved analytically, therefore one has to use numerical schemes. One way is to consider the s.d.e. directly. An alternative way is to investigate and solve the Fokker-Planck equation.

In this paper we study stochastic beam dynamics in twodimensional phase space. We describe a finite difference scheme to solve the corresponding Fokker-Planck equation with two phase space variables plus time. Extensive numerical simulations for this kind of problem have been performed in [3] using finite elements for the partial differential equation and Monte Carlo simulations for the s.d.e.. Here we concentrate on the finite difference scheme because of its simple implementation, its flexibility with respect to different boundary conditions, the straightforward extension to higher dimensional problems, its efficiency concerning CPU time and because it is easy to understand the physical meaning of each term in the scheme.
The paper is organized as follows: in section 2 we present the numerical scheme for solving the FokkerPlanck equation in $(2+1)$ variables and in section 3 we present some examples and applications to accelerator physics. Section 4 summarizes the main results and gives a list of open questions for future studies.

\section{NUMERICAL SCHEME FOR SOLVING THE FOKKER-PLANCK EQUATION}

We restrict our investigation to stochastic dynamics in twodimensional phase space. The general equations of motion we want to study are of the form

$$
\frac{d}{d s} x_{1}=x_{2}
$$

$$
\frac{d}{d s} x_{2}=-a_{1}\left(x_{1}\right)-a_{2}\left(x_{1}, x_{2}\right)+a_{3}\left(x_{1}\right) \eta_{1}+a_{4} \eta_{2}
$$

with $\eta_{1}, \eta_{2}$ a Gaussian white noise vector process. $a_{1}\left(x_{1}\right)$ can be an arbitrary nonlinear potential (field), $a_{2}\left(x_{1}, x_{2}\right)$ can include van der Pol-like damping terms, $a_{3}\left(x_{1}\right) \eta_{1}$ describes random parameters and $a_{4} \eta_{2}$ represents an additive noise term. The corresponding (Ito) Fokker-Planck equation for the probability density reads [2]

$$
\begin{aligned}
\frac{\partial}{\partial s} p & \left(x_{1}, x_{2}, s\right)= \\
& =-\frac{\partial}{\partial x_{1}}\left[x_{2} \cdot p\left(x_{1}, x_{2}, s\right)\right]+ \\
& +\frac{\partial}{\partial x_{2}}\left[\left(a_{1}\left(x_{1}\right)+a_{2}\left(x_{1}, x_{2}\right)\right) \cdot p\left(x_{1}, x_{2}, s\right)\right]+ \\
& +\frac{1}{2} \frac{\partial^{2}}{\partial x_{2}^{2}}\left[\left(a_{3}^{2}\left(x_{1}\right)+a_{4}^{2}\right) \cdot p\left(x_{1}, x_{2}, s\right)\right] .
\end{aligned}
$$

Equation (3) can be written in the form of two fluxes, one in $x_{1}$ and one in $x_{2}$ such that

$$
\frac{\partial p}{\partial s}=\frac{\partial A}{\partial x_{1}}+\frac{\partial B}{\partial x_{2}}
$$

a form which suggests to use an operator splitting method [4]. First we evaluate implicitly the $x_{2}$ derivative and then also implicitly - the $x_{1}$ derivative with a tridiagonal scheme

$$
\begin{aligned}
& \frac{p_{i, j}^{n+\frac{1}{2}}-p_{i, j}^{n}}{\Delta s}=\frac{F_{i, j+\frac{1}{2}}^{n+\frac{1}{2}}-F_{i, j-\frac{1}{2}}^{n+\frac{1}{2}}}{\Delta x_{2}} \\
& \frac{p_{i, j}^{n+1}-p_{i, j}^{n+\frac{1}{2}}}{\Delta s}=-x_{2} \frac{p_{i+1, j}^{n+1}-p_{i-1, j}^{n+1}}{2 \Delta x_{1}}
\end{aligned}
$$




$$
\begin{aligned}
& F_{i, j+\frac{1}{2}}=D \frac{p_{i, j+1}-p_{i, j}}{\Delta x_{2}}+ \\
& \quad+\left[a_{1}\left(x_{1}\right)+a_{2}\left(x_{1}, x_{2}+\Delta x_{2}\right)\right] \frac{p_{i, j+1}-p_{i, j}}{2}
\end{aligned}
$$

where we have set $2 D=\left[a_{3}^{2}\left(x_{1}\right)+a_{4}^{2}\right]$. Making the von Neumann analysis of stability it turns out that the scheme is unconditionally stable. For more information see [5],[6].

\section{RESULTS AND EXAMPLES}

\subsection{Harmonic oscillator}

The first example we have studied is the damped harmonic oscillator with strong diffusion i.e. $a_{1}\left(x_{1}\right)=K x_{1}$, $a_{2}\left(x_{1}, x_{2}\right)=\gamma x_{2}, a_{3}\left(x_{1}\right)=0$ and $a_{4}=\sigma . K, \gamma, \sigma$ are constants. Since this problem can be studied analytically [7] it served to check our numerical scheme. With the parameter set $K=1, \gamma=2.1, \sigma=0.8$, a $80 \times 80$ grid, $\Delta x_{1}=\Delta x_{2}=0.1, \Delta s=\pi / 1000$ and the exact solution at $s=0.95$ as initial condition we obtained the time evolution of the probability density depicted in figure 1 .

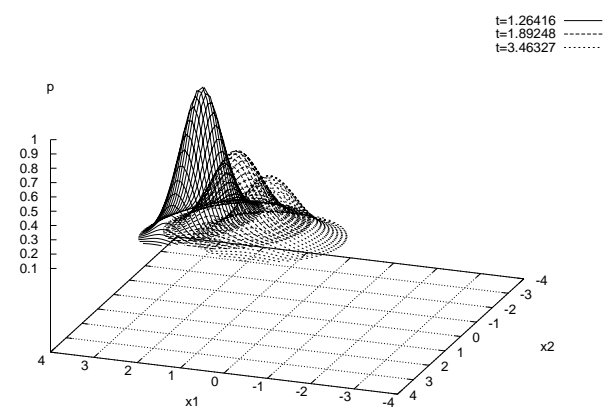

Figure 1: Density evolution in the damped linear harmonic oscillator equation, drift and diffusion resulting from the stochastic excitation.

\subsection{Duffing oscillator}

As a second example we have investigated the Duffing oscillator with damping, multiplicative and additive Gaussian white noise. In this case $a_{1}\left(x_{1}\right)=\omega^{2}\left[\alpha x_{1}+\epsilon x_{1}^{3}\right]$, $a_{2}\left(x_{1}, x_{2}\right)=2 \tau \omega x_{2}, a_{3}\left(x_{1}\right)=-\omega^{2} \sqrt{2 D_{11}} x_{1}$, and $a_{4}=$ $\sqrt{2 D_{22}} \cdot \omega=1, \alpha=-1, \tau=0.2, \epsilon=0.1$ are constants. For two different sets of noise intensities $D_{11}, D_{22}$ we obtained the results shown in figure 2 and figure 3 .

In this nonlinear system with multiplicative noise one can observe a noise induced transition [8]. In the additive noise case the system has two stable points just like the deterministic system. Under the influence of multiplicative noise the probability density at the origin grows. Further increasing of the strength of the multiplicative noise changes the stability of the origin - the origin becomes a stable point.

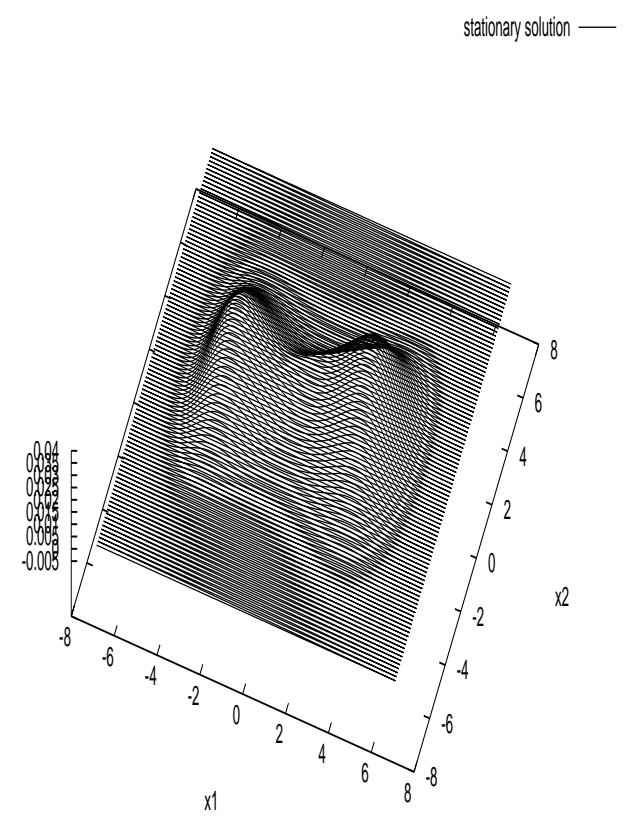

Figure 2: $D_{22}=0.4, D_{11}=0.08$, view of the stationary density in phase space. $\mathrm{dt}=\pi / 1000$ Finite difference integration.

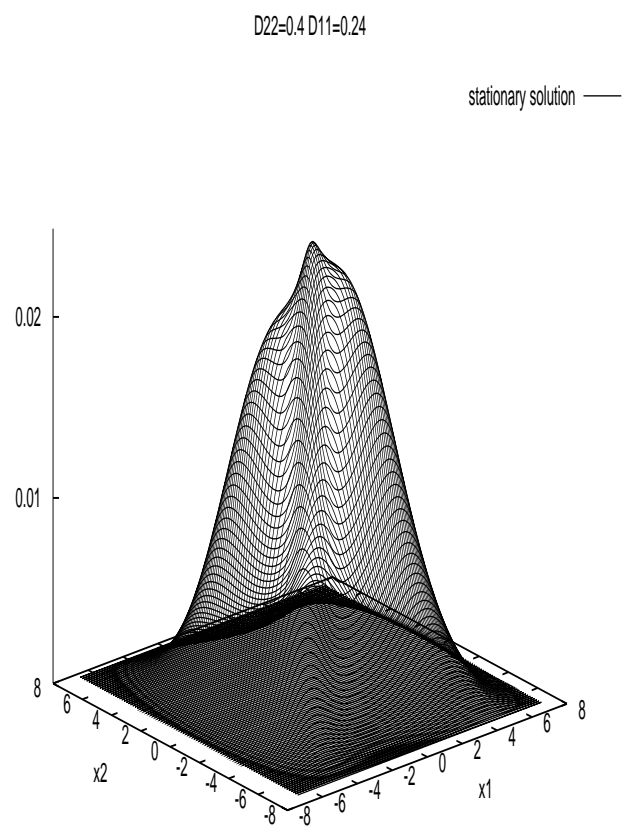

Figure 3: $D_{22}=0.4, D_{11}=0.24$, view of the stationary density in phase space. $\mathrm{dt}=\pi / 1000$ Finite difference integration.

\subsection{Beam-beam interaction in storage rings under the in- fluence of noise}

We have made a preliminary study of the the beam-beam interaction in storage rings under the influence of noise. 
The model we have chosen was defined by

$$
\begin{array}{r}
\frac{\partial p}{\partial s}=-\frac{\partial\left(x_{2} p\right)}{\partial x_{1}}+ \\
\frac{\partial}{\partial x_{2}}\left[\left(\alpha x_{2}+w^{2} x_{1}+f\left(x_{1}, s\right)\right) p\right]+\frac{1}{2} \frac{\partial^{2}}{\partial x_{2}^{2}}(d p) .
\end{array}
$$

with $\left.f\left(x_{1}, s\right)=\sum_{n=1}-8 \pi \xi_{b b}\left(\frac{1-e^{-\frac{1}{2} x_{1}^{2}}}{x_{1}}\right) \delta_{(} s=n L\right)$. We set the parameters to $\frac{d}{2}=0.000147, \tau=0.0000310, w=$ $0.7, \xi_{b b}=0.07$ (see figure 4 ).

Beam-beam effect $Q=3.7,100$ turns
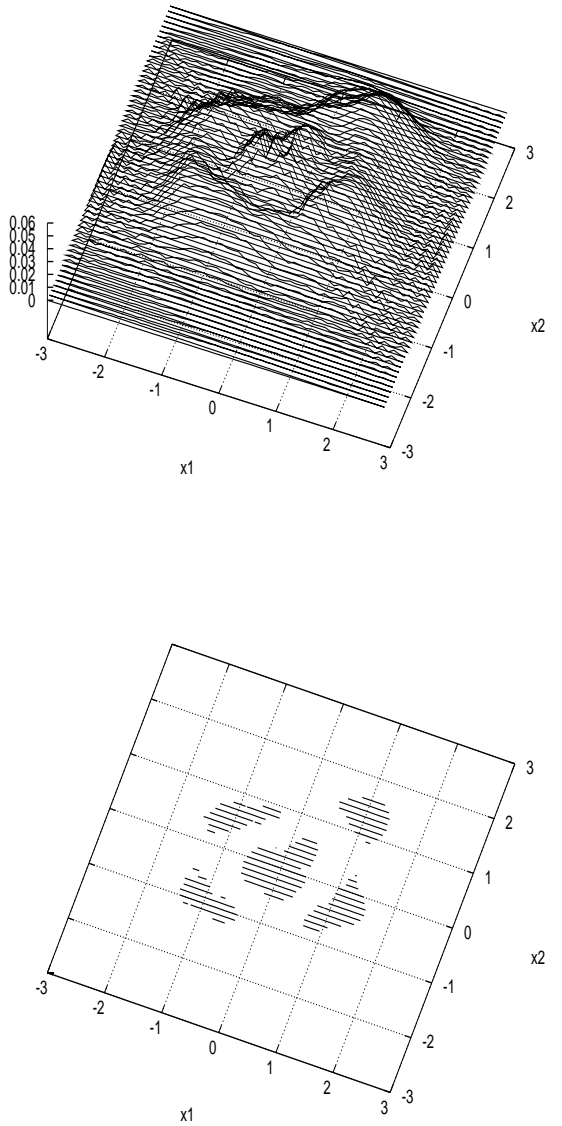

Figure 4: Electron case. Density for the beam-beam effect with $\mathrm{Q}=3.7, \xi=0.07$, damping time 250 turns. The lower figure is the projection onto the phase space of the areas of highest probability.

\section{SUMMARY AND CONCLUSIONS}

In this paper we have presented a robust finite difference scheme for the numerical solution of the Fokker-Planck equation in $(2+1)$ variables. The scheme has been checked with finite element calculations and with direct numerical simulations of the underlying s.d.e. It seems to be a good candidate for solving more complicated and more realistic accelerator models such as higher dimensional problems (coupled betatron motion), non- Gaussian white noise perturbations of Ornstein-Uhlenbeck type and explicitly time dependent coefficients $a_{i}(\ldots, s)$.

\section{ACKNOWLEDGEMENT}

This research was partly supported by the EC Human Capital and Mobility grant no: ERBCHRXCT940480, and by a DESY PhD scholarship. L.V. thanks the support of the Comision Interministerial de Ciencia y Tecnologia of Spain under grant PB95-0426. The authors want to thank L.A. Bergman, B.F. Spencer, S.F. Wojtkiewicz, E. Johnson for information about their work.

\section{REFERENCES}

[1] A. Pauluhn. Thesis: Stochastic Beam Dynamics in Storage Rings DESY 93-198 December 1993

[2] H. Risken. The Fokker-Planck Equation,Methods of Solutions and Applications. Springer-Verlag, 1984.

[3] B.F. Spencer, L.A. Bergman. Nonlinear Dynamics, 4 (1993) 357-372.

[4] W. Press, S. Teukolsky, W. Vetterling, B. Flannery. Numerical Recipes in C Cambridge University Press, 1994.

[5] R.D. Richtmyer, K.W. Morton. Difference Methods for Initial-Value Problems. Interscience Publishers, 1967.

[6] M.P. Zorzano, H. Mais, L. Vazquez. "Numerical solution of two dimensional Fokker-Planck equations" to be published.

[7] S. Chandrashekar. Stochastic Problems in Physics and Astronomy. Reviews of Modern Physics. Vol. 15. (1943) 1-44.

[8] W. Horsthemke, R. Lefever. Noise-Induced Transitions Theory and Applications in Physics, Chemistry, and Biology. Springer-Verlag Heidelberg New York Tokyo, 1984. 